cases to take extra precaution against a recurrence by fixing the uterus in its normal position, by shortening the round ligaments, or by ventral fixation. I should not by any means remove healthy ovaries when operating for retroflection by the section method. Should I find the ovaries diseased, then I would not hesitate to remove them. There is a proviso, however, that I wish to insert in this connection, namely: when the patient is young and would naturally wish to bear children, I should not then remove the ovaries, unless they were so altered by disease as to make fecundation impossible. In view of the fact that it is not always easy to define the extent of the destruction of normal ovarian stroma, we should give the would-be mother the benefit of all doubt and leave undisturbed the suspicious ovary. On the other hand, should the patient be the mother of several children-and there is no reason why she should have more children, unless she herself desires them, I should not be so particular to give the suspicious ovary a chance to assert its normal function, if to remove it would promise great relief to the sufferer.

From my no inconsiderable experience, I am prepared to make the statement, that it is only occasionally necessary to make the fine distinction before mentioned, for in nearly all cases of chronic retrodisplacements, the appendages are seriously involved and therefore diseased beyond any question.

The most satisfactory feature of the operation now under consideration, is the fact, almost universal, that the uterus will remain permanently in the anterior position without support, if the appendages are removed. It is surprising how emphatically nature asserts herself when the guy ropes are cut which hold the uterus in abnormal position. Immediately the uterus will go forward-almost with a bound-and reminds one of a sapling that has been bent to the ground and held, but which quickly returns to the upright position as soon as the force which binds it down is removed. So charming are the results of this operation in cases where the patient has suffered greatly for years, and has been subjected to all sorts of methods of treatment, and without permanent relief, until she has formed unfavorable opinions of the profession, that he who relieves her by this method is classed, and justly too, with the greatest of benefactors.

The next objection to this method, which I anticipate will be advanced is, that the danger to life is too great to operate in the abdomen for a condition which does not, of itself, destroy life, but only causes suffering and perhaps may do permanent harm only by establishing nervous sequelæ; this is the most important question to be considered in this connection. If it is a fact that the operation recommended is to result fatally, then of necessity we should only perform it as a dernier resort. But I contend that this operation should not give any greater mortality rate in properly selected cases than would amputation of a finger in properly selected cases. What I call properly selected cases, are those who have the endurance which will justify us in doing any kind of surgical operation of sufficient magnitude that complete anæsthesia and confinement in bed for two or three weeks would necessitate. That there is a degree of danger in any operation goes without saying and, therefore, it would be a mistake to assure the patient and friends that there is not some danger in this method; but it could be said with propriety that in the uncomplicated cases the mortality is about nil. Not more than 2 per cent. should perish. It is customary to fortify one's statements, when treating of a subject of this character by reporting cases, and I should gladly do so on this occasion, except for the reason that it is entirely unnecessary in view of the extensive experience of all present. Suffice it to say that there are a goodly number of ladies in my State who would gladly make testimony touching the renewed health-almost renewed life-they are enjoying; the result of this operation. No death has occurred to dampen my ardor, but I am always and at all times looking out for possible complications, by stating to the patient and friends that something may occur, and am on my guard, expecting something to occur that would cause all to grieve.

It is possible that I should have occupied more of the valuable time of this Section by a more detailed and elaborate classification of cases that should be subjected to abdominal section for the cure of displacements of the uterus; but I am obliged to leave to the generosity of my confrères to give the essayist the benefit of the doubt-as we would say in law-and convict him by trial before pronouncing judgment. In other words, I am not anxious to operate in the abdomen, if other methods less dangerous can be employed with good results.

\section{PROLAPSE OF THE FEMALE PELVIC ORGANS.}

Read in the Section of Obstetrics and Diseases of Women at the Fortyfourth Annual Meeting of the American Medical Association.

\section{BY HENRY PARKER NEWMAN, M.D.}

Prof. of Gynecology, Chicago Post-Graduate Medical School; Prof. Clinical Gynecology and Obstetrics. College of Physicians and

Surgeons; Gynecologist to Chicago Public and West Side Free Dispensaries; Physician and Surgeon to St. Elizabeth Hospital ; Surgeon, (department diseases of women) Chicago Post-Graduate Hos. pital; Secretary of the Chicago Gynecological Society: etc., etc.

It is my desire to call attention, briefly, to prolapse of the female genital organs, with particular reference to their hernial nature; regarding them as having a similar etiology, and the same pathological significance as herniæ in other situations, and amenable to analogous lines of treatment.

The classic division of herniæ into cephalic, thoracic and abdominal is incomplete without the important group comprised in the title of this paper; and, in view of their frequency and importance, they should enjoy an individual classification in herniology.

For accuracy and convenience, we would designate four major classes; cephalic, thoracic, abdominal and pelvic herniæ.

The etiology of these affections, in general, comprises such predisposing and exciting causes as defective development, including congenital malformations and inherited tendencies; depraved muscular tone through debilitating disease, and trauma.

While the majority of cases are generally attributed to traumatism, this may often in itself be the result of defective development, and inherited or acquired weaknesses or malformations.

Again, defective development represents not only the malformations of fœtal life, but the results of arrested growth at the period of ripening or puberty. 
Nature aims to furnish at birth suitable rudimentary pelvic organs, and it is exceedingly seldom that she fails to do so.

In the interference of artificial influences with the natural formative conditions of puberal activity, is to be found the proper explanation of many abnormalities of development.

It is an error to designate them as fcetal malformations. Under existing conditions of custom and education the female pelvis with its complex mechanism and its importance to the race, receives altogether insufficient recognition and conservation during puberty and adolescence.

Consequently, it is too poorly fortified against the demands made upon it during menstrual and childbearing epochs.

In the face of our boasted civilization and enlightenment, we must admit that the representative of budding womanhood who is addicted to corsets, heavy skirts and high-heeled shoes; lives on hot bread, pastry, pickles and sweetmeats; and, in our -cities, is in the highest grade at school, up six flights of stairs; and burns the midnight oil in the pursuit of knowledge or pleasure, is not the exception but the rule.

She is either ignorant of, or oblivious to the fact that she has an important set of generative organs which will probably be called upon one day to fulfill the purposes of their being, and that the proper performance of their future functions depends largely upon the judicious care bestowed upon the general health during the crises of development.

Rational treatment, therefore, of most gynecological diseases may begin before the affection has reached the surgical stage. This is particularly true of pelvic herniæ. An ounce of prophylaxis is worth a pound of pessaries.

The general practitioner, the family physician, has his opportanity before the specialist, and it is for jim, when occasion offers, to urge upon the parents nd guardians of young girls the necessity of attention to the regimen of the actively formative period of puberty.

Good food, sensible dress, a proper interchange of exercise and rest,-insist upon these things, general practitioners, for the sake of humanity's ultimate good, even if you take some bread out of the mouths of the specialists, into whose hands most neglected cases drift. The humanitarian gynecologist can well spare it. But it is not probable that his entire revenue from this source will be summarily cut off.

There is another factor in the production of pelvic disease which will furnish enough of its own victims to "keep hot the pathway to the office of the gynecologist," to quote an apt expression from a recent writer in a western journal. ${ }^{1}$

I refer to trauma in childbirth, and some of the prevalent evils of puerperal management, which render the normal process of reproduction a formidable menace to the after-health of the parous woman.

Many women whose puerperal histories go to make up some obstetrician's brilliant percentage of recoveries, have escaped (by the grace of God, and through no fault of the accoucheur,) with their lives, but with wrecked constitutions and dismantled pelves.

These cases, with subinvolution and torn pelvic floor, loss of tonicity of uterine and vaginal supports,

1 Neil Cameron, M.D., Omaha Clinic, March, '93. furnish frequent examples of all degrees of pelvic herniæ, and a strong argument in favor of preventive therapeutics.

In a former monograph I have referred to the excellent results to be expected from direct professional supervision over the entire period of gestation from the earliest months to the completion of labor.

But the consideration of prophylaxis in pregnancy, childbirth and the puerperal state is not the object of this paper.

I have wished simply to indicate its relation to downward displacements of the pelvic contents, and would emphasize the fact, once more, that it is easier to prevent such conditions than to remedy them.

Guard against the possibility of ruptures and lacerations during labor; repair at once such as are unavoidable; and use every effort to secure proper involution and a return of constitutional tone and muscular vigor, as far as is consistent with a normal puerperal state.

For the actual existence of pelvic herniæ,-cystocele, rectocele, prolapse of uterus and vaginal walls, prognosis and treatment depend upon the nature, degree and duration of the condition.

Much may be done in all cases, (minor cases of recent origin may sometimes be cured), by systemic treatment, attention to regimen, tonics, massage, faradism, local astringents, and removal of weight and constriction from the abdomen, with the view in all cases of encouraging the retentive power of its walls.

Proper action of the pelvic diaphragm is essential to the bealthful condition of the pelvic viscera.

This cannot be accomplished with constriction at the waist, or any impediment to free abdominal respiration.

All local treatment has for its aim the restoration of the prolapsed organ, and its retention in the normal position.

A hernia is a surgical disease and calls for surgical treatment, which admits of wide interpretation and includes all mechanical means from simple airpressure and gravitation in postural treatment to the radical operation of removal of the offending organ.

Simple restoration can usually be accomplished by taxis, preferable in the genupectoral position.

Exceptional cases, such as uterine prolapse with inversion, herniæ with adhesions or incarceration, may prove irreducible, and require special treatment, palliative or radical, as best suited to individual indications.

The retention of the replaced organ is not always an easy matter, and calls for great judgment in the seleetion of appropriate methods.

The simple tamponnade, combined with astringents and medicinal agents is extremely useful as a palliative measure, and in the treatment of complicating disorders, as chronic congestions, hyperplasias and inflammatory exudates.

But we can hardly expect permanently gratifying results from their use, except in recent and minor cases.

The same may be said of most other therapeutic agents and mechanical appliances, including the much-abused pessary.

The idea of both pessary and tampon is based upon the supposition of auxiliary pelvic support for its retention. 
This is, unfortunately, just what is lacking in most cases, and steps must be taken for its restoration. before either can be satisfactorily used.

The operation of perineorrhaphy, as done to-day is simple in technique, quickly performed, and efficacious in restoring the perineal body, and even where the laceration is complete is rarely followed by any but good results, if done for rectocele, or prolapse of the posterior vaginal wall.

In the same way the various colporrhaphies are most excellent in their sphere, that of reducing the volume of relaxed and prolapsed vaginal walls, but we mistake the pathology when we regard colpor. rhaphy as quite analogous to perineorrhaphy.

The latter removes the cause of the affection for which it is done, while the former may or may not do so.

In one instance we operate upon a substantial body, capable of sustaining the prolapsed viscus which had escaped by reason of the laxity or laceration of that body: while in the other, we have to deal with simple mucous membrane which, sooner or later, must again yield to the same opposing forces, unless the cause be removed.

It is even more fallacious to expect simple constricting of the vagina to remedy a hernia of the uterus, without any attempt to restore the anatomical relations of the parts, particularly the correct angle of uterine and vaginal axes.

A large and heavy uterus, with its abdominal supports weakened and stretched, and fundus and cervix perpendicular to the pelvic canal, will soon burrow its way through the cicatricial vaginal tissue by its own weight, supplemented by the vis-a-tergo of intra-abdominal pressure.

This tendency can be happily overcome by bringing the fundus forward into its normally anteverted position.

The three most serviceable operations to secure this result are, shortening of the utero-sacral ligaments; abdominal fixation of the fundus, or ventrofixation; and shortening of the round ligaments.

The latter, which is known as the AlexanderIdams operation has more to recommend it, in my judgment, than any other procedure.

It is founded upon the principle not of suspending the uterus, but of using the round ligaments as guy ropes to steady the organ in its normal anteverted direction, and bring it into auxiliary relation with the natural retentive forces of the abdomen.

The operation is simple of accomplishment, may often be done without general anæsthesia, and has given me such uniformly eatisfactory results that I heartily recommend it to those who desire to master its improved technique, which I have already detailed in former publications.

In conclusion, I would re-emphasize these points:

1. Pelvic hernias should be recognized and classified as such, and not as diseases of the uterus and adnexæ; and their treatment should be based upon hernial pathology.

2. Prophylaxis, in the formative stage of puberty, as well as in pregnancy and labor, is of the utmost moment in this class of cases.

3. Operations upon the pelvic floor or vaginal walls, while indispensable in their place cannot be relied upon alone to cure all herniæ of the pelvic viscera.

4. The ideal treatment is to be found in the com bination of operations for repair, and those for accessory support from above, and these may all be accomplished at the same sitting, saving the delay and annoyance of repeated operations.

Venetian Building, Chicago.

\section{VESICO-VAGINAL FISTULA; ITS ETIOLOGY AND TREATMENT.}

Read before the Section of Obstetrics and Diseases of Women, at the Forty-fourth Annual Meeting of the American Medical Associution.

BY AUgustus P. CLARKE, A.M., M.D. CAMBRIDGE. MASS.

Vesico-vaginal fistula may be described as a preternatural opening between the bladder and the vagina. The communication may be located at the cervix or at the fundus of the bladder. Sometimes an opening from the urethra into the vagina takes place; an opening thus established is usually smaller and less jagged than is one situated in the neck or in the fundus of that viscus. Urethro-vaginal fistulæ may result from the compression to which the parts. are exposed during parturition. It will be observed that the urethra in its oblique descent under the arch of the pubes toward the meatus urinarius occupies for some distance the anterior portion of the vaginal wall; this position exposes the urethro-vaginal tissue to many accidents during a difficult or tedious parturient process.

In those cases in which the fistula is situated in a higher segment of the bladder, sloughing that has often caused an opening may have been superinduced or hastened by labor. Many conditions which are dependent on pregnancy favor the development of fistula; such conditions predispose to disease the vesical tissues which finally require only the super. vention of parturition for an actual occurrencle of the morbid process.

An acute cystitis may come on during the courf of pregnancy; in such cases there may be an ex tended vascular injection of the mucous tissue. Brownish or discolored patches may occur in the vicinity of the neck and fundus of the bladder; there will occur at first more or less induration or alteration of the vesical walls, and later there will be present a fibrinous and purulent exudation at different foci or in areas in which serious and degenerative changes have taken place. The disease may be limited to the mucous lining of the bladder; in cases in which there may be some renal complication the morbid process may penetrate the layers of the muscular coat and involve the peritoneum posteriorly in its upper zone or may implicate the vaginal wall anteriorly in its lower section. Suppuration may be dependent on the presence of minute abscesses occurring in the parietes of the bladder; ulcerative processes may also have so continued as to have led to softening, maceration, commencing gangrene or destruction of portions of the mucous and subjacent muscular structures.

Not long since I was called to an autopsy of a woman aged 37 years; she had died of acute pneumonia complicated with other serious troubles. There was found a degenerative change which had taken place midway between the fundus and the neck of the bladder. The mucous, the connective and the muscular plains in limited areas were observed almost entirely destroyed; the integrity of 